\title{
A numerical method for incompressible non-Newtonian fluid flows based on the lattice Boltzmann method
}

\author{
M. Yoshino ${ }^{\text {a,*}}$, Y. Hotta $^{\text {a,** }}{ }^{\text {, T. }}$ Hirozane $^{\text {a }}$, M. Endo $^{\text {b }}$ \\ ${ }^{a}$ Department of Mechanical Systems Engineering, Shinshu University \\ 4-17-1 Wakasato, Nagano 380-8553, Japan \\ ${ }^{\mathrm{b}}$ Department of Electrical and Electronic Engineering, Shinshu University \\ 4-17-1 Wakasato, Nagano 380-8553, Japan
}

\begin{abstract}
A new numerical method for incompressible non-Newtonian fluid flows based on the lattice Boltzmann method (LBM) is proposed. The essence of the present method lies in the determination of shear-dependent viscosity of the fluid by using a variable parameter related to the local shear rate. Also, the relaxation time in the BGK collision term is kept at unity taking account of numerical stability. The method is applied to two representative test case problems, power-law fluid flows in a reentrant corner geometry and non-Newtonian fluid flows in a three-dimensional porous structure. These simulations indicate that the method can be useful for practical non-Newtonian fluid flows, such as shear-thickening (dilatant) and shearthinning (pseudoplastic) fluid flows.
\end{abstract}

Key words: Lattice Boltzmann method (LBM); Non-Newtonian fluids; Power-law fluids; Reentrant corner flow; Porous structure; Darcy's law 


\section{Introduction}

During the last two decades, the lattice Boltzmann method (LBM) [1-5] has been developed into an alternative and promising numerical scheme for simulating viscous fluid flows and multicomponent and multiphase fluid flows (see reviews [6-9]). In particular, the LBM has been successfully applied to various kinds of complex flows mainly for Newtonian fluids. On the other hand, non-Newtonian fluid flows are also of great importance in many science and engineering applications, such as complex flows containing surfactants and/or colloids $[10,11]$ and plastic flows including Carbon fiber $[12,13]$ in industrial devices.

One of the advantages of the LBM is that the shear tensor can be computed locally, with no need of taking space derivatives of the velocity field [14]. Hence, the LBM is considered to offer excellent possibilities for simulating non-Newtonian fluid flows. As for previous studies, Aharonov and Rothman [15] first introduced a lattice Boltzmann model for power-law fluids [16], which are non-Newtonian fluids based on the power-law model. Rakotomalala et al. [17] proposed an LB model for non-Newtonian shear-thickening and shearthinning fluids whose viscosity depends on local shear rate. Giraud et al. $[18,19]$ also developed two- and three-dimensional LB models of simple viscoelastic fluid flows. Then, Boek et al. [20] showed the validity of the early model by Aharonov and Rothman in two-dimensional fundamental flow problems. Gabbanelli et al. [21] also proposed similar but another LBM for the truncated power-law fluids by setting lower and upper cut-off values of the

* Corresponding author. Tel: +81-26-269-5116; fax: +81-26-269-5116.

**Present address: Tokai Rika Co. Ltd., Aichi 480-0195, Japan

Email address: masato@shinshu-u.ac.jp (M. Yoshino). 
fluid viscosity. More recently, Sullivan et al. [22] have extended such LBM to a three-dimensional model, and applied it to non-Newtonian flow problems in a porous structure.

In most of the above-mentioned techniques, a relaxation time in the BGK collision term is varied as a function of the shear stress at each time step to give the correct local viscosity. Indeed, such practices of varying the relaxation time are very common in LB modeling of turbulent flows (e.g. see Ref. [23]) as well as of non-Newtonian fluid flows. However, the relaxation time has influence on the numerical stability of the LB scheme, that is, the LBM with the BGK model becomes unstable for the relaxation time close to $1 / 2[24,25]$ (corresponding to small viscosities). In particular, for non-Newtonian fluid flows, the relaxation time is also related to the local shear rate, so that numerical instability can occur despite relatively large zero-shear-rate viscosity (which is the viscosity when the shear rate tends to zero). To avoid this difficulty, for example, Gabbanelli et al. set the lower and upper bounds on the viscosity in Ref. [21]. For practical use, however, it is desirable to develop a new LB model which is applicable in a wide range of shear-dependent viscosity.

In recent years, Inamuro [26] has proposed the lattice kinetic scheme for Newtonian viscous fluid flows as an extension scheme of the LBM. In this scheme, the relaxation time is set to unity so that the numerical stability can be obtained for relatively high Reynolds number flows; nevertheless one is able to determine the fluid viscosity using a constant parameter appearing in the additional term of the equilibrium distribution function. Thus, taking advantage of such a heuristic approach, one can construct a new LB model for non-Newtonian fluid flows by regarding the constant parameter as a variable parameter dependent on the local shear rate. 
The paper is organized as follows. In Sec. 2 we give preliminary description of non-Newtonian fluids with shear-dependent viscosity. In Sec. 3 we propose a numerical method for incompressible non-Newtonian fluid flows based on the LBM. In Sec. 4 we investigate the appropriateness and accuracy of the method in a channel flow. We present numerical examples, flow in a reentrant corner geometry and flow in a three-dimensional porous structure, in Sec. 5. In two problems, flow characteristics and local shear rate are calculated. Also, in the latter problem the relation between pressure drops and fluid flux is examined. Finally, concluding remarks are given in Sec. 6 .

\section{Background}

An incompressible viscous fluid is assumed in the present study. For a nonNewtonian fluid, effective viscosity $\mu$ is found to vary with local shear rate $\dot{e}$. Here the shear rate is related to the second invariant of the symmetric strain rate tensor $e_{\alpha \beta}$ as follows:

$$
\dot{e}=\sqrt{e_{\alpha \beta} e_{\alpha \beta}}
$$

with

$$
e_{\alpha \beta}=\frac{1}{2}\left(\frac{\partial u_{\beta}}{\partial x_{\alpha}}+\frac{\partial u_{\alpha}}{\partial x_{\beta}}\right)
$$

where $\boldsymbol{u}$ is the fluid velocity, and subscripts $\alpha$ and $\beta$ represent Cartesian coordinates and the summation convention is used hereafter.

A commonly used model for non-Newtonian fluids is the power-law, or Ostwaldde Waele model [16], and they are often referred to as power-law fluids. The effective viscosity of the power-law fluids is given by 


$$
\mu=\mu_{1}|\dot{e}|^{(n-1)}=\mu_{1}\left|e_{\alpha \beta} e_{\alpha \beta}\right|^{(n-1) / 2} \quad \text { for } n>0
$$

where $n$ is the power-law exponent and $\mu_{1}$ is a proportional constant. Note that the case where $n=1$ is a Newtonian fluid, in which $\mu_{1}$ corresponds to the viscosity of the Newtonian fluid. For a fluid with $n>1$, the effective viscosity increases with shear rate, and the fluid is called shear-thickening or dilatant fluid. For a fluid with $0<n<1$, the effective viscosity decreases with shear rate, and the fluid is called shear-thinning or pseudoplastic fluid.

Another model used for shear-thinning fluids is the Carreau model [27], where the effective viscosity is expressed by

$$
\frac{\mu-\mu_{\infty}}{\mu_{0}-\mu_{\infty}}=\left[1+(\lambda \dot{e})^{2}\right]^{(n-1) / 2} \quad \text { for } 0<n \leq 1
$$

where $\mu_{0}$ is the zero-shear-dependent viscosity $(\dot{e} \rightarrow 0), \mu_{\infty}$ is the infinityshear-dependent viscosity $(\dot{e} \rightarrow \infty)$, and $\lambda$ is the time constant. The value of $\mu_{\infty}$ is often set to zero for simplicity.

\section{Numerical method}

Hereafter, we use non-dimensional variables defined by a characteristic length $H$, a characteristic particle speed $c$, a characteristic time scale $t_{0}=H / U$ where $U$ is a characteristic flow speed, and a reference density $\rho_{0}[9]$. In the LBM, a modeled fluid composed of identical particles whose velocities are restricted to a finite set of $N$ vectors $\boldsymbol{c}_{i}(i=1,2, \ldots, N)$ is considered. Although the 15-velocity model $(N=15)$ is used in the following descriptions, they can be directly applied to other velocity models. The velocity vectors in the 15velocity model are given by 


$$
\begin{aligned}
& {\left[\boldsymbol{c}_{1}, \boldsymbol{c}_{2}, \boldsymbol{c}_{3}, \boldsymbol{c}_{4}, \boldsymbol{c}_{5}, \boldsymbol{c}_{6}, \boldsymbol{c}_{7}, \boldsymbol{c}_{8}, \boldsymbol{c}_{9}, \boldsymbol{c}_{10}, \boldsymbol{c}_{11}, \boldsymbol{c}_{12}, \boldsymbol{c}_{13}, \boldsymbol{c}_{14}, \boldsymbol{c}_{15}\right]} \\
& \quad=\left[\begin{array}{rrrrrrrrrrrrrrr}
0 & 1 & 0 & -1 & 0 & 0 & 0 & 1 & -1 & -1 & 1 & 1 & -1 & -1 & 1 \\
0 & 0 & 1 & 0 & -1 & 0 & 0 & 1 & 1 & -1 & -1 & 1 & 1 & -1 & -1 \\
0 & 0 & 0 & 0 & 0 & 1 & -1 & 1 & 1 & 1 & 1 & -1 & -1 & -1 & -1
\end{array}\right]
\end{aligned}
$$

Here, we introduce the lattice kinetic scheme, which is an extension method of the original LBM [26]. In this method, the physical space is divided into a cubic lattice, and the evolution of macroscopic variables, fluid density $\rho$ and fluid velocity $\boldsymbol{u}$, at the lattice point $\boldsymbol{x}$ and at time $t$ are defined as follows:

$$
\begin{aligned}
& \rho(\boldsymbol{x}, t)=\sum_{i=1}^{15} f_{i}^{\mathrm{eq}}\left(\boldsymbol{x}-\boldsymbol{c}_{i} \Delta x, t-\Delta t\right), \\
& \boldsymbol{u}(\boldsymbol{x}, t)=\frac{1}{\rho(\boldsymbol{x}, t)} \sum_{i=1}^{15} \boldsymbol{c}_{i} f_{i}^{\mathrm{eq}}\left(\boldsymbol{x}-\boldsymbol{c}_{i} \Delta x, t-\Delta t\right),
\end{aligned}
$$

where $\Delta x$ is a spacing of the cubic lattice, and $\Delta t$ is a time step which is chosen so that $\Delta t=\operatorname{Sh} \Delta x$ where $\mathrm{Sh}=U / c$ is the Strouhal number. Also, $f_{i}^{\text {eq }}$ is given by $[5,26]$

$$
\begin{aligned}
f_{i}^{\mathrm{eq}}=E_{i} \rho\left[1+3 c_{i \alpha} u_{\alpha}+\frac{9}{2} c_{i \alpha} c_{i \beta} u_{\alpha} u_{\beta}\right. & -\frac{3}{2} u_{\alpha} u_{\alpha} \\
& \left.+A \Delta x\left(\frac{\partial u_{\beta}}{\partial x_{\alpha}}+\frac{\partial u_{\alpha}}{\partial x_{\beta}}\right) c_{i \alpha} c_{i \beta}\right]
\end{aligned}
$$

where $E_{1}=2 / 9, E_{2}=E_{3}=\cdots=E_{7}=1 / 9, E_{8}=E_{9}=\cdots=E_{15}=1 / 72$ with $\alpha, \beta=x, y, z$, and $A$ is described below. The pressure $p$ is related to the density by

$$
p=\frac{1}{3} \rho
$$

In this scheme, macroscopic variables are calculated without velocity distribution functions. Thus, the scheme can save memory because there is no need 
to store the velocity distribution functions. Moreover, according to Ref. [26], test calculations show that the scheme is more stable than the original LBM. Therefore, the lattice kinetic scheme based on the LBM is suited to development of the new method for non-Newtonian fluid flows.

For Newtonian fluids, $A$ in Eq. (8) is a constant parameter of $O(1)$ which determines a constant viscosity of the fluid. For non-Newtonian fluids, on the other hand, $A$ is considered to be a variable parameter which depends on the local shear rate as follows. Applying the asymptotic theory [28,29] to Eqs. (6) and (7) with (8), the viscosity $\mu$ of the fluid is obtained by

$$
\mu=\left(\frac{1}{6}-\frac{2}{9} A\right) \Delta x
$$

Hence, for power-law fluids, $A$ is specified as follows:

$$
A=\frac{3}{4}-\frac{9}{2} \mu_{1} \dot{e}^{(n-1)}(\Delta x)^{-1}
$$

The same approach can be applied to other non-Newtonian fluids. In the case of Carreau modeled fluids, for example, $A$ is given by

$$
A=\frac{3}{4}-\frac{9}{2} \mu_{\infty}(\Delta x)^{-1}-\frac{9}{2}\left(\mu_{0}-\mu_{\infty}\right)\left[1+(\lambda \dot{e})^{2}\right]^{(n-1) / 2}(\Delta x)^{-1}
$$

The first derivatives of the velocity appearing in the above equations can be approximated by, for instance,

$$
\frac{\partial u_{\beta}}{\partial x_{\alpha}} \approx \frac{1}{10 \Delta x} \sum_{i=2}^{15} c_{i \alpha} u_{\beta}\left(\boldsymbol{x}+\boldsymbol{c}_{i} \Delta x\right)
$$

Using the Taylor series expansion, it is easily found that Eq. (13) is an approximation by the second-order central difference. 
It should be noted that in the present method, boundary conditions for the macroscopic variables are the same as those in usual computational fluid dynamics (CFD) methods. For example, at stationary walls, $\boldsymbol{u}=\mathbf{0}$ is directly given as the no-slip boundary condition.

\section{Appropriateness and accuracy}

To verify the appropriateness and accuracy of the method, we calculate nonNewtonian fluid flows between parallel walls. Here we use the two-dimensional nine-velocity model $(N=9)$ for simplicity. The nine-velocity model has the following velocity vectors: $\boldsymbol{c}_{1}=\mathbf{0}, \boldsymbol{c}_{i}=[\cos (\pi(i-2) / 2), \sin (\pi(i-2) / 2)]$ for $i=$ $2,3,4,5$, and $\boldsymbol{c}_{i}=\sqrt{2}[\cos (\pi(i-11 / 2) / 2), \sin (\pi(i-11 / 2) / 2)]$ for $i=6,7,8,9$. The basic idea and formulation for the nine-velocity model are the same as those for the 15-velocity model except that the values of the constants $E_{i}$ are $E_{1}=4 / 9, E_{2}=E_{3}=E_{4}=E_{5}=1 / 9$, and $E_{6}=E_{7}=E_{8}=E_{9}=1 / 36$.

A steady flow between stationary parallel walls at $y= \pm 1 / 2$ with a constant pressure gradient is calculated for power-law fluids. A periodic boundary condition with pressure difference is used at the inlet and outlet, and a no-slip boundary condition is employed on the walls. In the case of power-law fluids, the exact solution of the velocity normalized by the mean flow velocity is given by $[16]$

$$
u_{x, \text { exa }}^{*}(y)=\frac{u_{x, \text { exa }}}{\bar{u}_{x, \text { exa }}}=\frac{2 n+1}{n+1}\left[1-|2 y|^{(1+1 / n)}\right]
$$

where $n$ is the power-law exponent, and the subscript 'exa' and the superscript '*' indicate exact solution and normalized value, respectively. 
We first carry out simulations for power-law fluids with $n=0.5,0.75,1.25$, and 2 by setting $\Delta x=1 / 40$. The calculations are repeated until the following convergence criterion is satisfied:

$$
\frac{\left|u_{x}(\boldsymbol{x}, t)-u_{x}(\boldsymbol{x}, t-\Delta t)\right|}{\left|u_{x}(\boldsymbol{x}, t)\right|}<\delta
$$

for all $u_{x}(\boldsymbol{x}, t) \geq 0.01 \times \max \left\{\left|u_{x}(\boldsymbol{x}, t)\right|\right\}$ where the maximum is searched over the whole domain. The tolerance $\delta$ is set to $1 \times 10^{-8}$ in this problem. The parameter related to viscosity $\mu_{1}$ is varied between $1.1 \times 10^{-3} \Delta x$ and $2.2 \times 10^{3} \Delta x$. The pressure difference between the inlet and outlet is fixed at $\Delta p=1 \times 10^{-4}$. Fig. 1 shows the calculated velocity profiles normalized by the mean flow velocity for various values of $n$. The result for a Newtonian fluid $(n=1)$ is also shown. In addition, the exact solution obtained by Eq. (14) for each $n$ is drawn by a solid line. It is found that the results for $0.5 \leq n \leq 2$ agree fairly well with the exact solution.

We next perform the same simulations for $\Delta x=1 / 10,1 / 20,1 / 40$, and $1 / 100$, and calculate errors of the numerical results from the exact solution. The error norm is defined by

$$
\operatorname{Er}=\frac{\sum_{y}\left|u_{x}^{*}-u_{x, \text { exa }}^{*}\right|}{\sum_{y}\left|u_{x, \text { exa }}^{*}\right|}
$$

It should be noted that the sums in Eq. (16) are taken over the common 11 positions at $y= \pm j / 10(j=0,1,2,3,4,5)$ in all cases. The calculated error norm is shown in Fig. 2. The slopes indicating the convergence rate are $1.981,2.002,1.998$, and 1.872 for $n=0.5,0.75,1.25$, and 2 , respectively. These mean that the method is an almost second-order scheme, though small deviation is found in the result for $n=2$. It is also found that for $\Delta x=1 / 100$ 
the magnitude of every error in our simulations is less than $0.1 \%$. Moreover, in comparison with results by Gabbanelli et al. [21], the present method is more accurate than their method; this is especially true of the cases for the shear-thinning fluids $(n=0.5,0.75)$. In fact, at the highest lattice resolution $(\Delta x=1 / 100)$, the ratios of the errors in the present work to those in Ref. [21] are $1 / 18,1 / 27,1 / 5$, and $1 / 10$ for $n=0.5,0.75,1.25$, and 2.0, respectively. One reason for their larger errors would be that they set the lower and upper cut-off values of the viscosity in order to avoid numerical instability. Thus, their results of velocity near the walls slightly deviate from the exact solution due to the limited range of viscosity.

\section{Numerical examples}

\subsection{Flow in a reentrant corner geometry}

In contrast to the previous unidirectional flow, we now consider a more demanding geometry, namely, the reentrant corner geometry presented in Fig. 3. The domain is divided into square lattice so that $L=80 \Delta x$. Initial conditions are $\rho=1$ and $\boldsymbol{u}=\mathbf{0}$ in the whole domain. The periodic boundary condition with pressure difference is used at the inlet and outlet, and the no-slip boundary condition is used on the walls. The parameter $\mu_{1}$ ranges from $2 \times 10^{-3} \Delta x$ to $2 \times 10^{-1} \Delta x$. Other parameters are fixed at $\Delta p=1 \times 10^{-5}$ and $\delta=1 \times 10^{-6}$. In this problem, the convergence criterion defined as the relative velocity difference in Sec. 4 is applied to the vertical component as well as the horizontal component, and the calculations are repeated until both criteria are satisfied.

Fig. 4 shows flow fields for different power-law exponents $n=0.5,0.75$, and 
1. It should be noted that all arrows are drawn by an uniform length only for visualization of flow direction. In fact, the magnitude of fluid velocity inside the cavity is from 30 to 80 times smaller than that of the velocity in the channel. Steady circular flow can be seen in the cavity region for all cases, and the core of the circulation shifts toward the channel and slightly toward the inlet, as $n$ becomes smaller. Next, Fig. 5 shows the contour plot of the local magnitude of shear rate. It is seen that the shear rate is very large around the entrant and reentrant corners, which are both singular points. Fig. 6 shows the velocity profiles of $u_{x} / \bar{u}_{x}$ on the line $x / L=0.50$ and of $u_{y} / \bar{u}_{x}$ on the line $y / L=0.40$. Note that the enlargement in the region of $0.60 \leq y / L \leq 1$ is also given in the inset. In Fig. 6 (a), plug-like velocity profiles which are characteristic of shear-thinning fluids can be seen especially for $n=0.5$. In Fig. 6 (b), there is some fluid penetration into the cavity, i.e., entrant flow in the first half of the channel and some reentrant flow in the second half. Also, the flow fields are not symmetric about $x / L=0.50$ owing to convection effects. These phenomena are in good agreement with the results by Gabbanelli et al. $[21]$.

\subsection{Flow in a three-dimensional porous structure}

As an example of three-dimensional problems, power-law fluid flows in a porous structure shown in Fig. 7 is considered. There exist rectangular bodies in a cubic domain with the sides of length $L$. The domain is divided into $64 \times 64 \times 64$ lattice. The porosity of the structure is $\varepsilon=0.75$. Initial conditions are $\rho=1$ and $\boldsymbol{u}=\mathbf{0}$ in the whole domain. The periodic boundary condition with pressure difference is assumed at the inlet and outlet. A normal periodic 
boundary condition is applied to the other sides of the domain. Also, the noslip boundary condition is used on the bodies. The parameters are changed in the range of $4.4 \times 10^{-3} \Delta x \leq \mu_{1} \leq 2.2 \times 10^{-1} \Delta x$ and $1 \times 10^{-6} \leq \Delta p \leq 1 \times 10^{-3}$ for different power-law exponents. The same convergence criterion as Eq. (15) is used with $\delta=1 \times 10^{-6}$.

Fig. 8 shows the calculated velocity vectors (left) and shear rate distribution (right) on different planes $(y / L=0.375, z / L=0.375$, and $x / L=0.375)$ for $\Delta p=1 \times 10^{-3}$ and $n=0.75$, after transitional flows. For comparison, the same simulation is carried out for a Newtonian fluid, and the corresponding results are illustrated in Fig. 9. Then the Reynolds number $\operatorname{Re}=\rho u_{0} L / \mu_{1}$ where $u_{0}$ is the superficial velocity becomes 0.262 , which belongs to the laminar flow region. For the non-Newtonian fluid in Fig. 8, flow characteristics near the bodies are influenced by the local shear rate. The shear rate distribution is much more complicated and shows very steep gradients in the direction perpendicular to the mainstream around the bodies. For the Newtonian fluid in Fig. 9, on the other hand, parabola-like velocity profiles appear in the pore regions between the bodies, and flow patters around each body are found to be symmetric. Also, the shear rate is almost uniform and quite different from the results for the non-Newtonian fluid. Next, the relation between pressure drops and fluid flux is examined. The fluid flux $q$ is defined as the volumetric flow rate divided by the void area; it follows that the flux is equal to the superficial velocity. In the laminar flow region, the generalized Darcy's law $[16,30,31]$ is valid for power-law fluids flowing through porous media:

$$
q=K\left(\frac{\Delta p}{L}\right)^{1 / n}
$$

where $K$ depends on the exponent $n$. Hence, the validation of the method is 
achieved by observing whether logarithmic plot of $q$ versus $\Delta p$ gives a straight line with slope of $1 / n$. Fig. 10 shows the relation between these quantities. The resultant slopes $(1 / n)$ are tabulated in Table 1 . It is seen that each error is less than $2 \%$ compared to the generalized Darcy's law. Although the present simulations yield accurate results with $\Delta x=1 / 64$, higher lattice resolution would be required for more complicated porous structures.

\section{Concluding remarks}

We propose a numerical method for incompressible non-Newtonian fluid flows based on the LBM. In the simulation of power-law fluid flows between parallel walls, the calculated velocity profiles are in good agreement with theory for the power-law exponents of $0.5 \leq n \leq 2$. The error analysis is carried out and the method is more accurate than the standard approach of making the relaxation time a function of local shear rate. Using the present method, we simulate power-law fluid flows in a reentrant corner geometry and in a three-dimensional porous structure. In addition, simulations of non-Newtonian Carreau modeled fluids are performed and similar results are obtained for such problems, though they are not shown in the paper. Therefore, it is expected that the method will be a promising numerical scheme for simulating non-Newtonian fluid flows in a wide range of shear-dependent viscosities.

Finally, in the problem of flows through porous structures, the accuracy of the method would depend on the porosity $\varepsilon$ and the lattice spacing $\Delta x$ as well as geometric structure of porous media. The study of the accuracy concerning these parameters remains in future work. 


\section{Acknowledgments}

The authors would like to thank a reviewer for valuable comments and helpful suggestions on the manuscript. This research was supported by the Grant-inAid (No. 18760121) and by the CLUSTER of Ministry of Education, Culture, Sports, Science and Technology, Japan. 


\section{Nomenclature}

A parameter determining a fluid viscosity

c characteristic particle speed $[\mathrm{m} / \mathrm{s}]$

$\boldsymbol{c}_{i} \quad$ particle velocity

$e_{\alpha \beta} \quad$ strain rate tensor

$\dot{e} \quad$ shear rate

$E_{i} \quad$ constants in an equilibrium distribution function

Er error norm

$f_{i}^{\text {eq }} \quad$ equilibrium distribution function

$H \quad$ characteristic length $[\mathrm{m}]$

$K$ proportional coefficient related to permeability

$L \quad$ length of domain

$n \quad$ power-law exponent

$N \quad$ number of particle velocity vectors

$p \quad$ pressure

$q \quad$ fluid flux

Re Reynolds number, $\rho u_{0} L / \mu_{1}$

Sh Strouhal number, $U / c$

$t \quad$ time

$t_{0} \quad$ characteristic time scale $[\mathrm{s}]$

$\boldsymbol{u}$ fluid velocity, $\left(u_{x}, u_{y}\right)$ or $\left(u_{x}, u_{y}, u_{z}\right)$

$u_{0} \quad$ superficial velocity

$\bar{u}_{x} \quad$ mean flow velocity

$U \quad$ characteristic flow speed $[\mathrm{m} / \mathrm{s}]$

$\boldsymbol{x} \quad$ Cartesian coordinates, $(x, y)$ or $(x, y, z)$ 
Greek letters

$\delta \quad$ tolerance of convergence criterion

$\Delta p \quad$ pressure difference between inlet and outlet

$\Delta t \quad$ time step

$\Delta x \quad$ lattice spacing

$\varepsilon \quad$ porosity

$\lambda \quad$ time constant

$\mu \quad$ effective viscosity

$\mu_{0} \quad$ zero-shear-dependent viscosity

$\mu_{1} \quad$ viscosity of a Newtonian fluid

$\mu_{\infty} \quad$ infinity-shear-dependent viscosity

$\rho \quad$ density

$\rho_{0} \quad$ reference density $\left[\mathrm{kg} / \mathrm{m}^{3}\right]$

Subscripts

exa exact solution

$\alpha \quad$ Cartesian coordinates

$\beta \quad$ Cartesian coordinates

$x \quad x$-component

$y \quad y$-component

Superscripts

* normalized

eq equilibrium 


\section{References}

[1] G.R. McNamara, G. Zanetti, Use of the Boltzmann equation to simulate latticegas automata, Phys. Rev. Lett. 61 (1988) 2332-2335.

[2] F. Higuera, S. Succi, R. Benzi, Lattice gas dynamics with enhanced collisions, Europhys. Lett. 9 (1989) 345-349.

[3] F. Higuera, J. Jimenez, Boltzmann approach to lattice gas simulations, Europhys. Lett. 9 (1989) 663-668.

[4] H. Chen, S. Chen, W.H. Matthaeus, Recovery of the Navier-Stokes equations using a lattice-gas Boltzmann method, Phys. Rev. A 45 (1992) 5339-5342.

[5] Y.H. Qian, D. d'Humières, P. Lallemand, Lattice BGK models for NavierStokes equation, Europhys. Lett. 17 (1992) 479-484.

[6] R. Benzi, S. Succi, M. Vergassola, The lattice Boltzmann equation: theory and applications, Phys. Rep. 222 (1992) 145-197.

[7] S. Chen, G.D. Doolen, Lattice Boltzmann method for fluid flows, Annu. Rev. Fluid Mech. 30 (1998) 329-364.

[8] S. Succi, The Lattice Boltzmann Equation for Fluid Dynamics and Beyond, Oxford University Press, Oxford, 2001.

[9] T. Inamuro, Lattice Boltzmann methods for viscous fluid flows and for twophase fluid flows, Fluid Dyn. Res. 38 (2006) 641-659.

[10] P.M. Vlahovska, M. Loewenberg, J. Blawzdziewicz, Deformation of a surfactantcovered drop in a linear flow, Phys. Fluids 17 (2005) 103103.

[11] J. Bergenholtz, J.F. Brady, M. Vicic, The non-Newtonian rheology of dilute colloidal suspensions, J. Fluid Mech. 456 (2002) 239-275. 
[12] M. Drubetski, A. Siegmann, M. Narkis, Hybrid particulate and fibrous injection molded composites: Carbon black/Carbon fiber/polypropylene systems, Polym. Compos. 26 (2005) 454-464.

[13] K. Araki, T. Kitano, A. Lengalova, P. Saha, Transient shear flow properties of carbon fiber-filled liquid crystalline polymer, Polym. Compos. 26 (2005) 470476.

[14] S. Ubertini, S. Succi, Recent advances of Lattice Boltzmann techniques on unstructured grids, Prog. Comput. Fluid Dyn. 5 (2005) 85-96.

[15] E. Aharonov, D.H. Rothman, Non-Newtonian flow (through porous media): a lattice-Boltzmann method, Geophys. Res. Lett. 20 (1993) 679-682.

[16] R.B. Bird, W.E. Stewart, E.N. Lightfoot, Transport Phenomena, John Wiley \& Sons, New York, 1960, Chapters 1 and 6.

[17] N. Rakotomalala, D. Salin, P. Watzky, Simulations of viscous flows of complex fluids with a Bhatnagar, Gross, and Krook lattice gas, Phys. Fluids 8 (1996) $3200-3202$.

[18] L. Giraud, D. d'Humières, P. Lallemand, A lattice-Boltzmann model for viscoelasticity, Int. J. Mod. Phys. C 8 (1997) 805-815.

[19] L. Giraud, D. d'Humières, P. Lallemand, A lattice Boltzmann model for Jeffreys viscoelastic fluid, Europhys. Lett. 42 (1998) 625-630.

[20] E.S. Boek, J. Chin, P.V. Coveney, Lattice Boltzmann simulation of the flow of non-Newtonian fluids in porous media, Int. J. Mod. Phys. B 17 (2003) 99-102.

[21] S. Gabbanelli, G. Drazer, J. Koplik, Lattice Boltzmann method for nonNewtonian (power-law) fluids, Phys. Rev. E 72 (2005) 046312.

[22] S.P. Sullivan, L.F. Gladden, M.L. Johns, Simulation of power-law fluid flow through porous media using lattice Boltzmann techniques, J. Non-Newtonian 
Fluid Mech. 133 (2006) 91-98.

[23] H. Chen, S. Kandasamy, S. Orszag, R. Shock, S. Succi, V. Yakhot, Extended Boltzmann kinetic equation for turbulent flows, Science 301 (2003) 633-636.

[24] J.D. Sterling, S. Chen, Stability analysis of lattice Boltzmann methods, J. Comput. Phys. 123 (1996) 196-206.

[25] X.D. Niu, C. Shu, Y.T. Chew, T.G. Wang, Investigation of stability and hydrodynamics of different lattice Boltzmann models, J. Stat. Phys. 117 (2004) 665-680.

[26] T. Inamuro, A lattice kinetic scheme for incompressible viscous flows with heat transfer, Philos. Trans. R. Soc. Lond. A 360 (2002) 477-484.

[27] R.B. Bird, R.C. Armstrong, O. Hassager, Dynamics of polymeric liquids, vol.1, Fluid Dynamics, 2nd ed., John Wiley \& Sons, New York, 1987.

[28] Y. Sone, Asymptotic theory of flow of rarefied gas over a smooth boundary II, in: D. Dini (Ed.), Rarefied gas dynamics, vol. 2, Pisa, Editrice Tecnico Scientifica, 1971, p. 737.

[29] T. Inamuro, M. Yoshino, F. Ogino, Accuracy of the lattice Boltzmann method for small Knudsen number with finite Reynolds number, Phys. Fluids 9 (1997) $3535-3542$.

[30] J.R.A. Pearson, P.M.J. Tardy, Models for flow of non-Newtonian and complex fluids through porous media, J. Non-Newtonian Fluid Mech. 102 (2002) 447473.

[31] A. Fadili, P.M.J. Tardy, J.R.A. Pearson, A 3D filtration law for power-law fluids in heterogeneous porous media, J. Non-Newtonian Fluid Mech. 106 (2002) 121146. 


\section{Figure captions}

Fig. 1. Velocity profiles between parallel walls for power-law fluids with $n=0.5,0.75,1.25$, and 2 . The result for a Newtonian fluid $(n=1)$ is also shown. $\bar{u}_{x}$ is the mean flow velocity. The solid lines represent the exact solution.

Fig. 2. Error norm of flow between parallel walls for power-law fluids. The closed and open symbols indicate results by the present study and by Gabbanelli et al. [21], respectively: circle, $n=0.5$; square, $n=0.75$; triangle, $n=1.25$; diamond, $n=2$.

Fig. 3. Two-dimensional reentrant corner geometry.

Fig. 4. Flow fields in reentrant corner geometry for different power-law exponents: (a) $n=0.5$; (b) $n=0.75$; (c) $n=1$. Note that all arrows are drawn by an uniform length only for visualization of flow direction.

Fig. 5. Contour plots of local magnitude of shear rate for different power-law exponents: (a) $n=0.5$; (b) $n=0.75$; (c) $n=1$.

Fig. 6. Velocity profiles: (a) horizontal component, $u_{x}$, on the line $x / L_{x}=$ 0.50 ; (b) vertical component, $u_{y}$, on the line $y / L_{y}=0.40 . \bar{u}_{x}$ is the mean flow velocity.

Fig. 7. Three-dimensional porous structure.

Fig. 8. Velocity vectors (left) and shear rate distribution (right) of power-law fluid with $n=0.75$ for $\Delta p=1 \times 10^{-3}$ : (a) on $y / L=0.375$; (b) on $z / L=0.375$; (c) on $x / L=0.375 . u_{0}$ is the superficial velocity. Contour interval of shear 
rate is $3.05 \times 10^{-5}$.

Fig. 9. Velocity vectors (left) and shear rate distribution (right) of Newtonian fluid for $\Delta p=1 \times 10^{-3}$ : (a) on $y / L=0.375$; (b) on $z / L=0.375$; (c) on $x / L=0.375 . u_{0}$ is the superficial velocity. Contour interval of shear rate is $3.05 \times 10^{-5}$.

Fig. 10. Relation between pressure drops and fluid flux of flows in threedimensional porous structure. 
Table 1

$1 / n$ slopes obtained from Fig. 10 of flows in three-dimensional porous structure

\begin{tabular}{lccc}
\hline$n$ & $1 / n$ & slope & error(\%) \\
\hline 1 & 1 & 1.000 & 0.001 \\
0.85 & 1.176 & 1.157 & 1.639 \\
0.75 & 1.333 & 1.308 & 1.898 \\
\hline
\end{tabular}




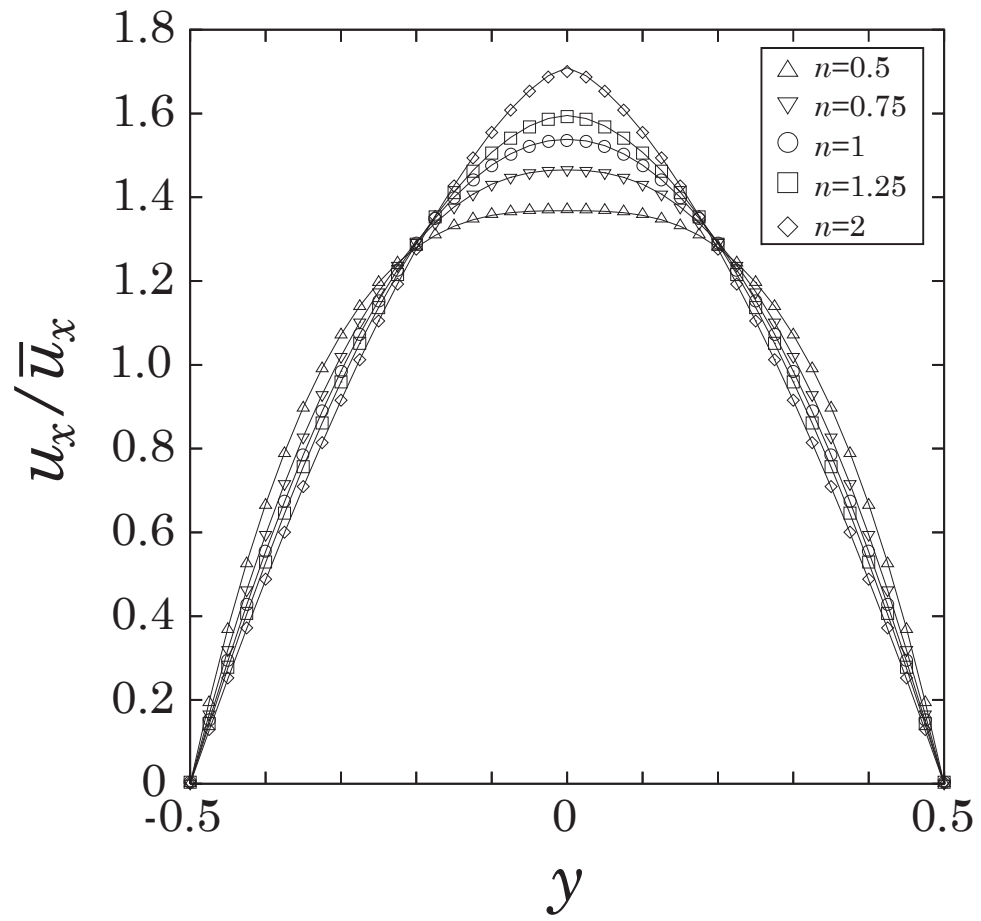

Fig. 1. Velocity profiles between parallel walls for power-law fluids with $n=0.5,0.75,1.25$, and 2 . The result for a Newtonian fluid $(n=1)$ is also shown. $\bar{u}_{x}$ is the mean flow velocity. The solid lines represent the exact solution. 


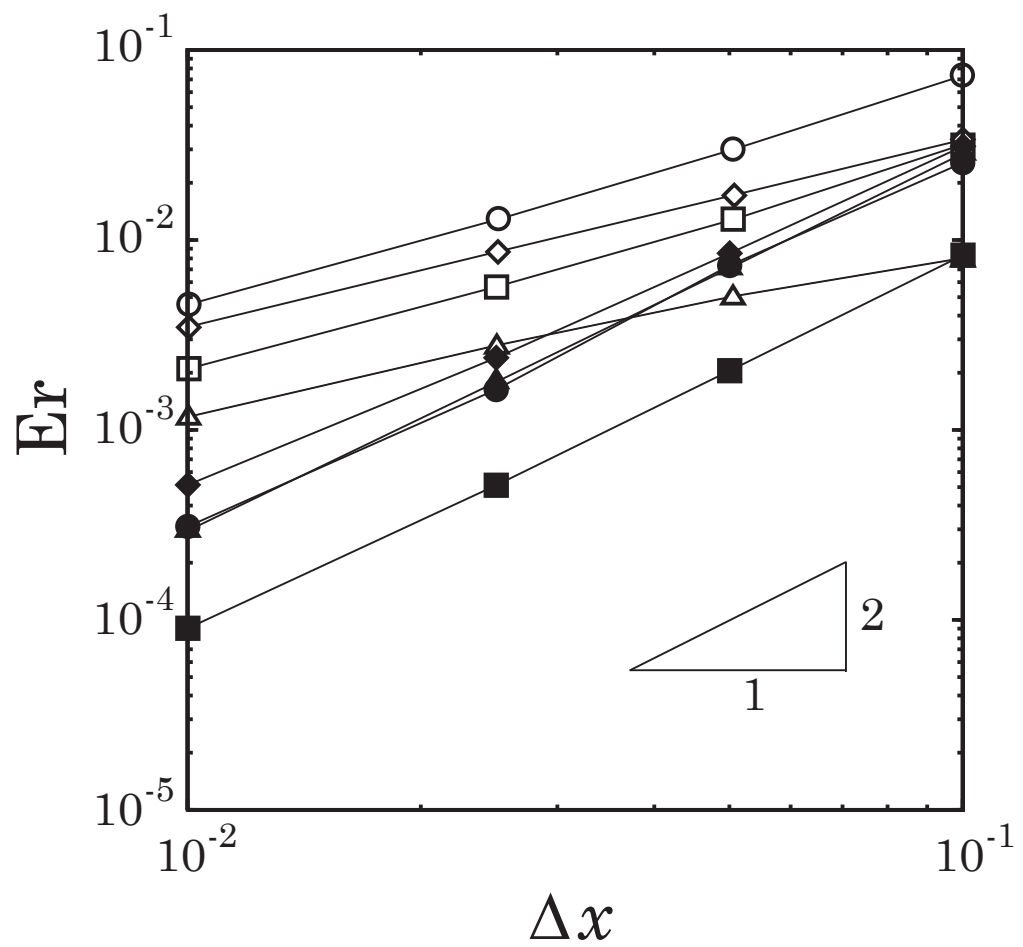

Fig. 2. Error norm of flow between parallel walls for power-law fluids. The closed and open symbols indicate results by the present study and by Gabbanelli et al. [21], respectively: circle, $n=0.5$; square, $n=0.75$; triangle, $n=1.25$; diamond, $n=2$. 


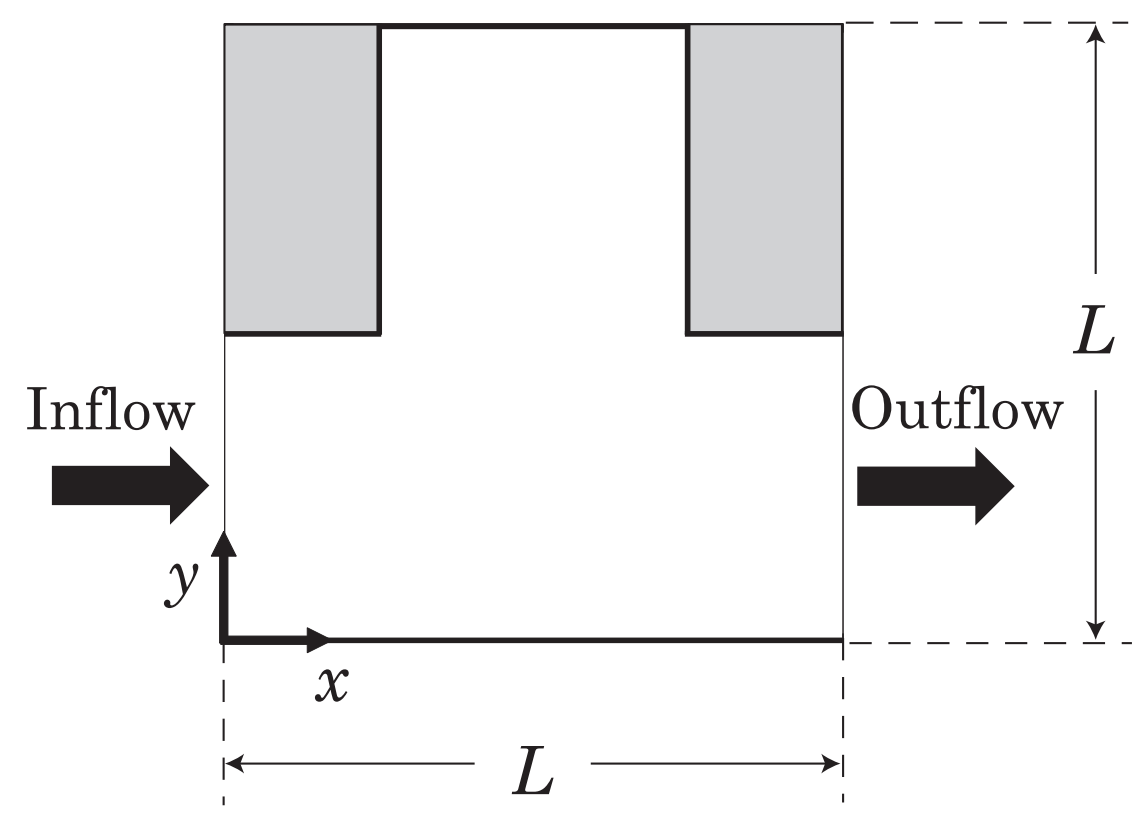

Fig. 3. Two-dimensional reentrant corner geometry. 


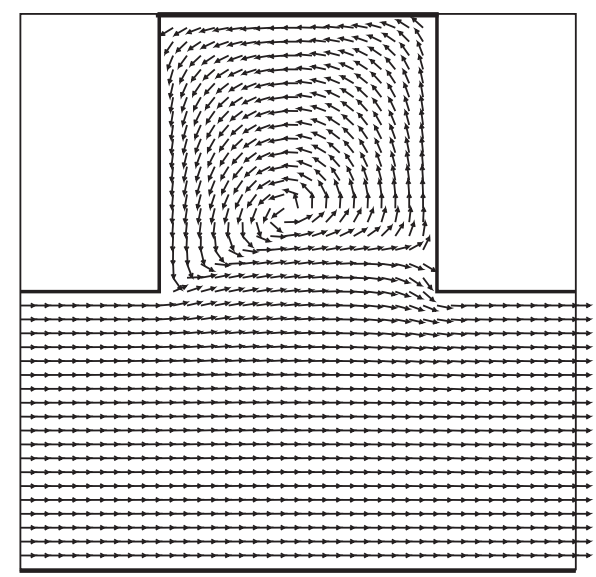

(a)

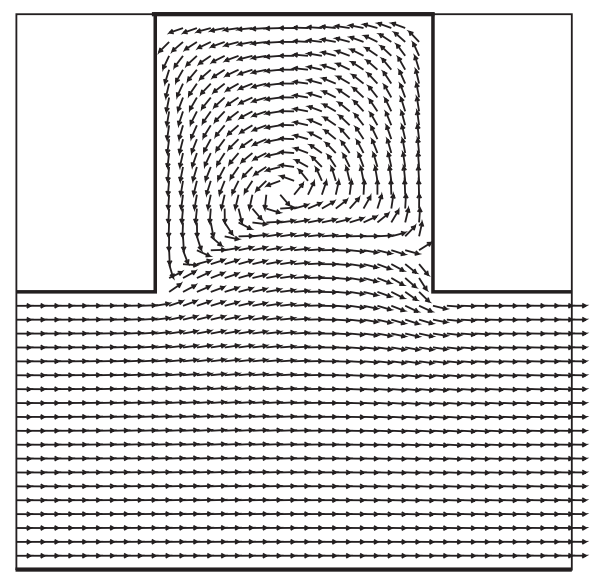

(b)

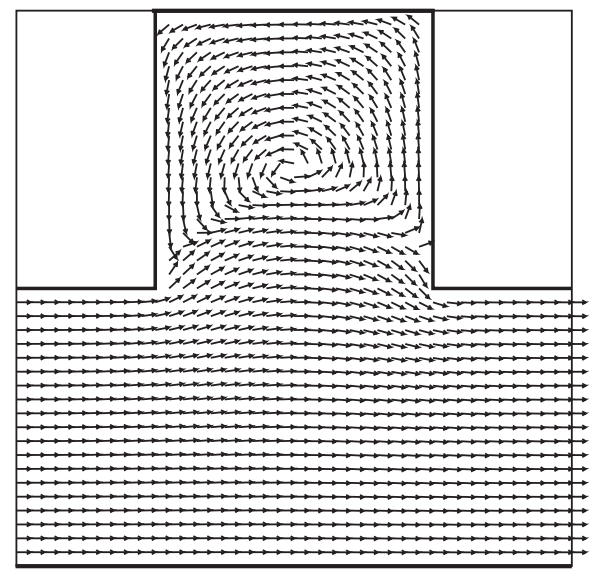

(c)

Fig. 4. Flow fields in reentrant corner geometry for different power-law exponents: (a) $n=0.5$; (b) $n=0.75$; (c) $n=1$. Note that all arrows are drawn by an uniform length only for visualization of flow direction. 


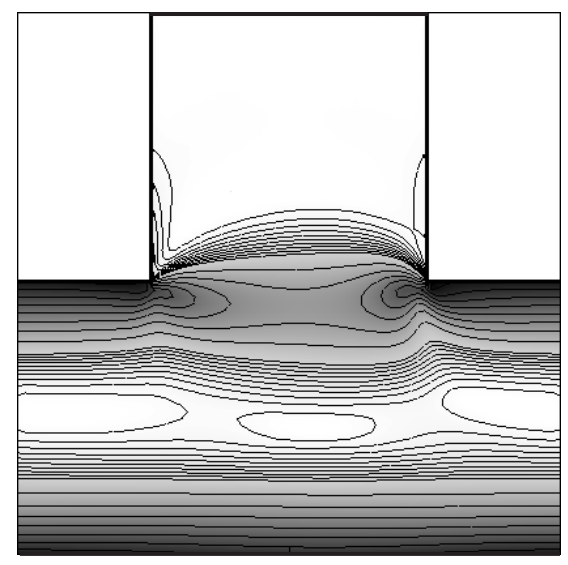

(a)

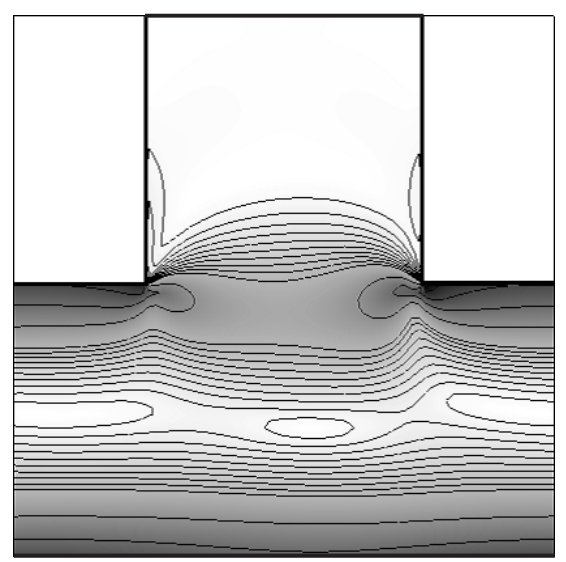

(b)
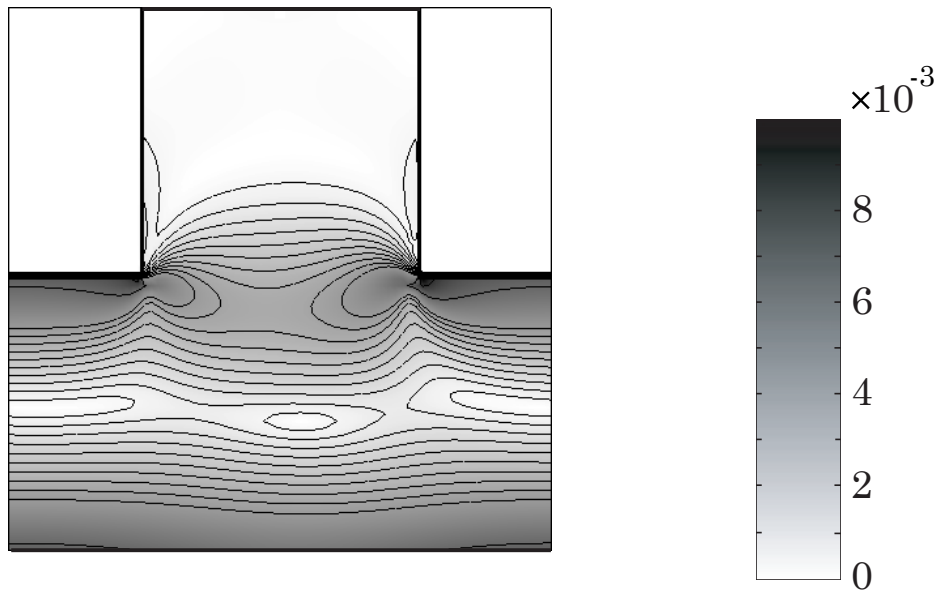

(c)

Fig. 5. Contour plots of local magnitude of shear rate for different power-law exponents: (a) $n=0.5$; (b) $n=0.75$; (c) $n=1$. 


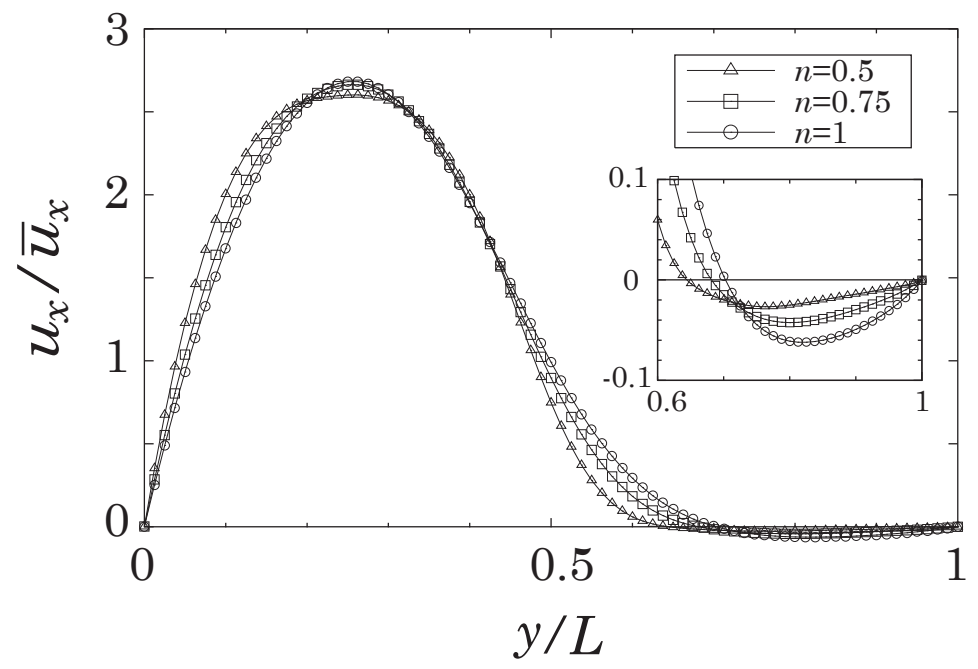

(a)

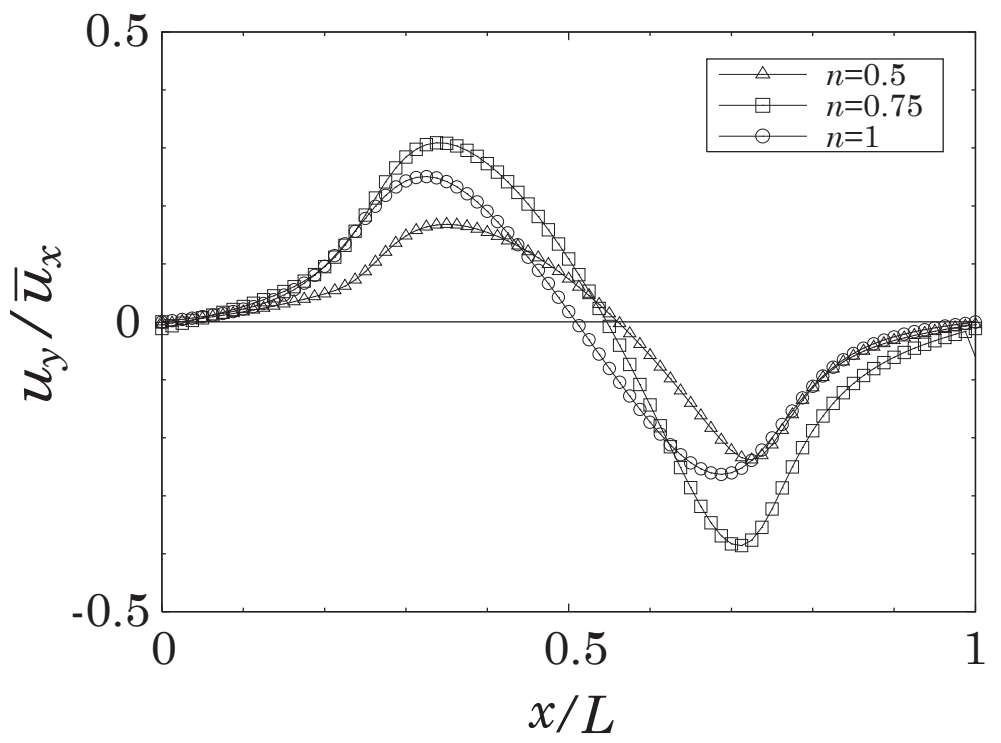

(b)

Fig. 6. Velocity profiles: (a) horizontal component, $u_{x}$, on the line $x / L_{x}=0.50$; (b) vertical component, $u_{y}$, on the line $y / L_{y}=0.40 . \bar{u}_{x}$ is the mean flow velocity. 


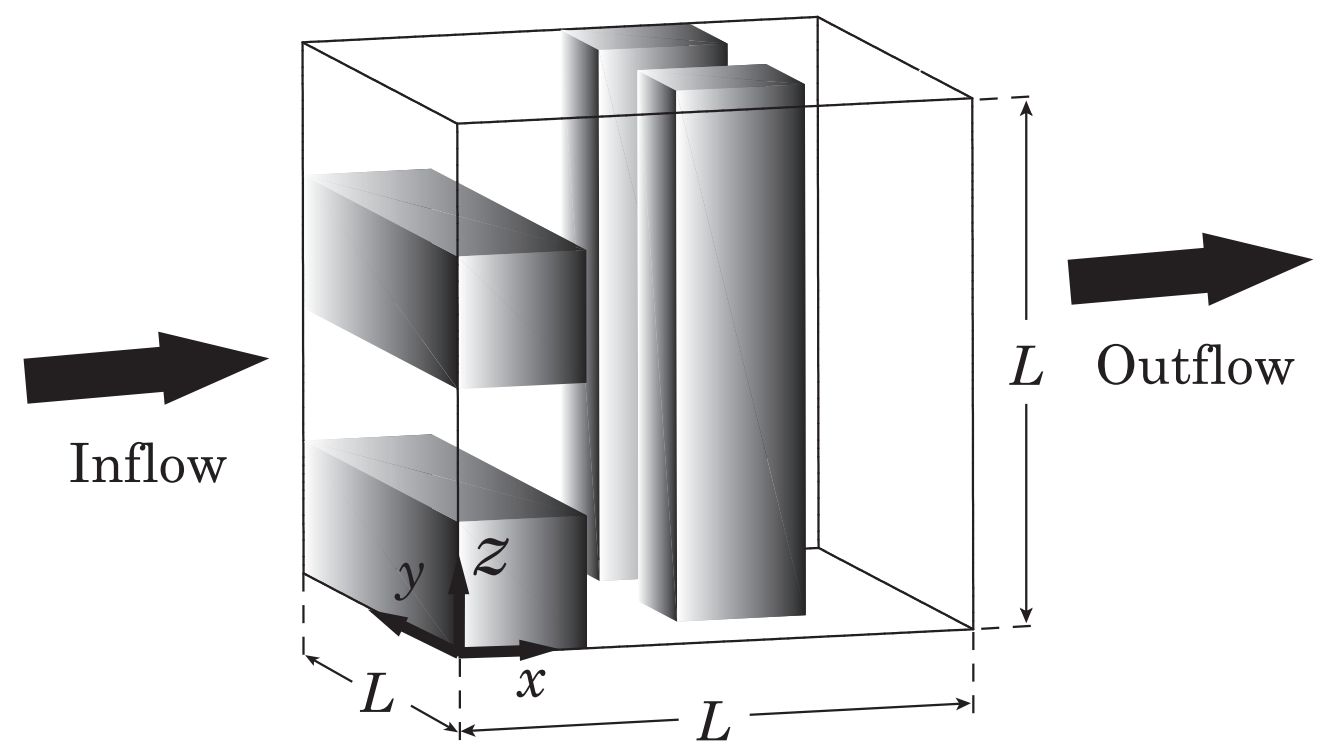

Fig. 7. Three-dimensional porous structure. 

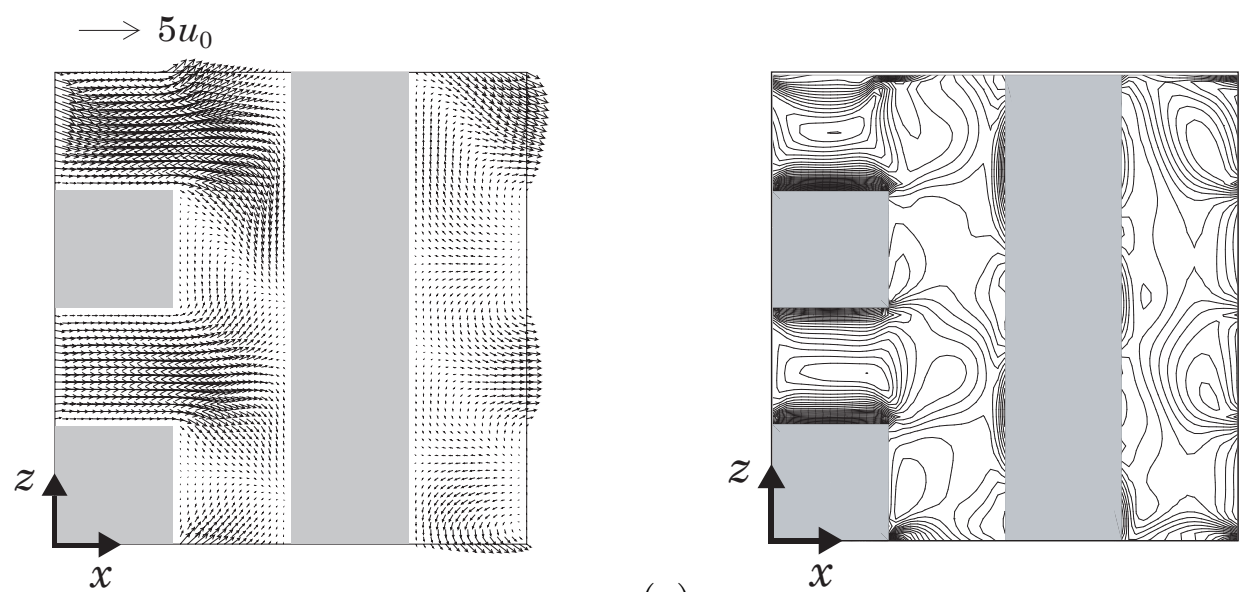

(a)

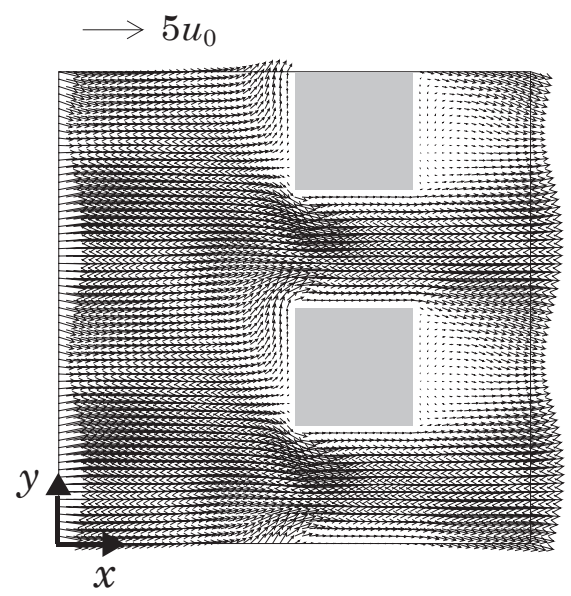

(b)

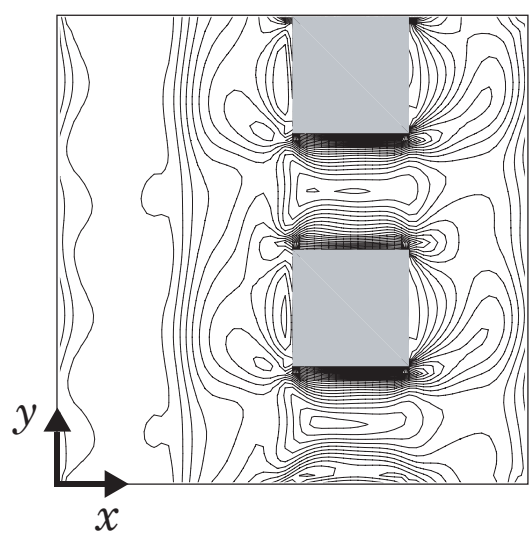

$\longrightarrow 5 u_{0}$

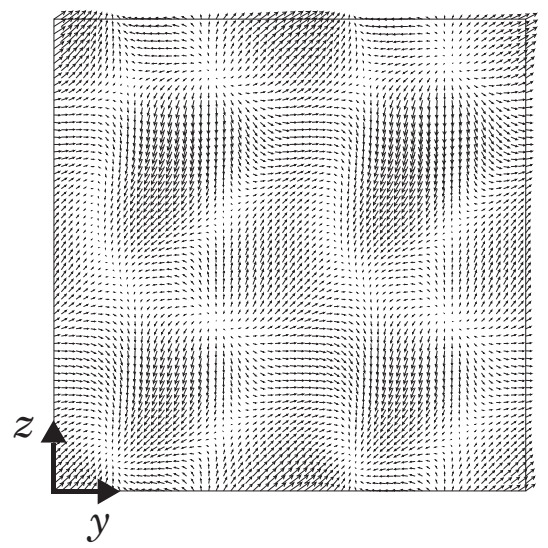

(c)

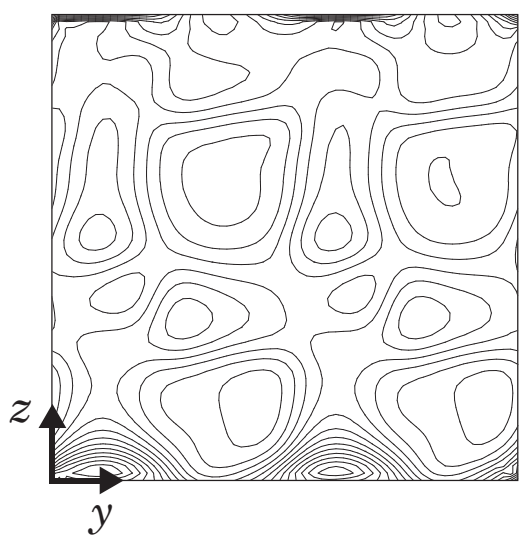

Fig. 8. Velocity vectors (left) and shear rate distribution (right) of power-law fluid with $n=0.75$ for $\Delta p=1 \times 10^{-3}$ : (a) on $y / L=0.375$; (b) on $z / L=0.375$; (c) on $x / L=0.375 . u_{0}$ is the superficial velocity. Contour interval of shear rate is $3.05 \times 10^{-5}$. 

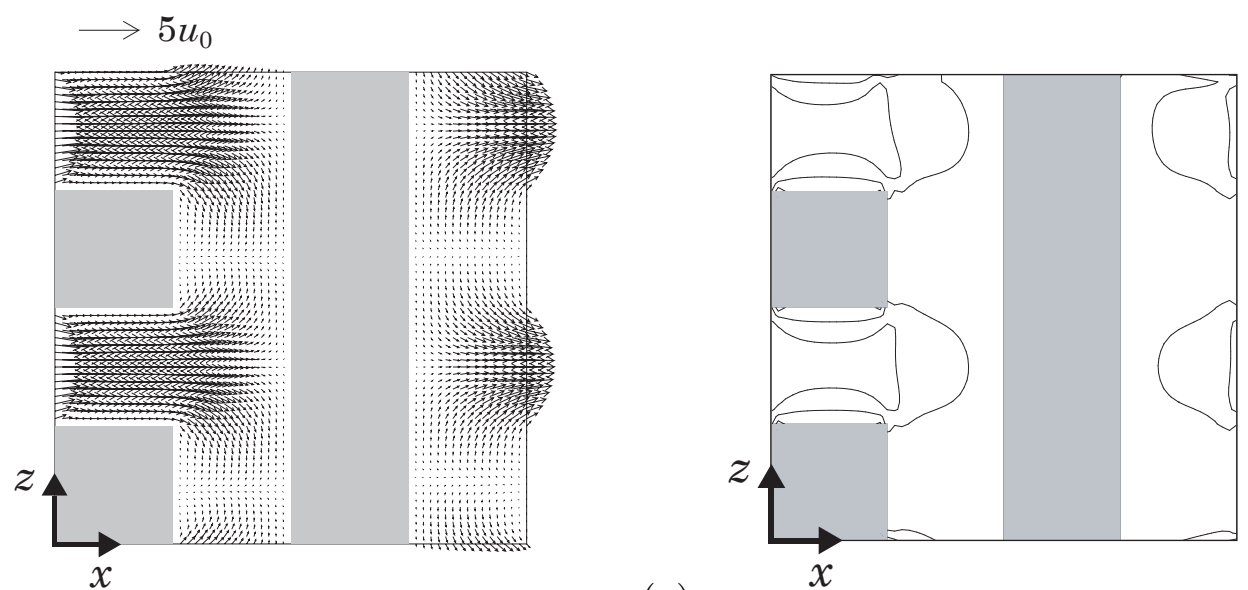

(a)

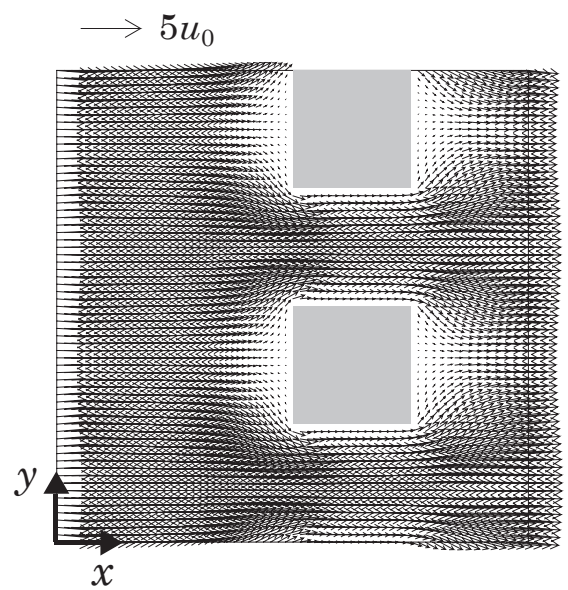

(b)
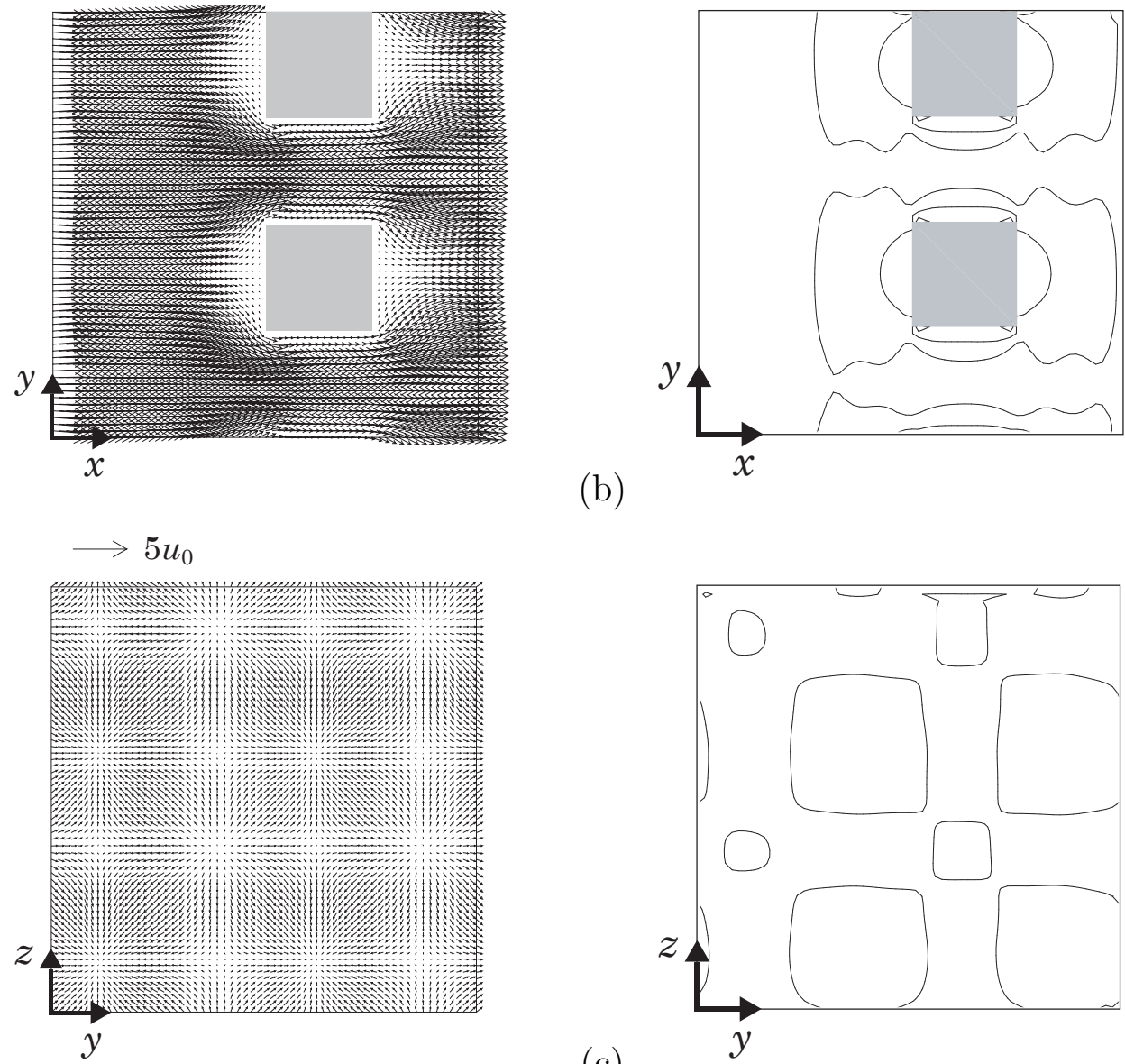

(c)

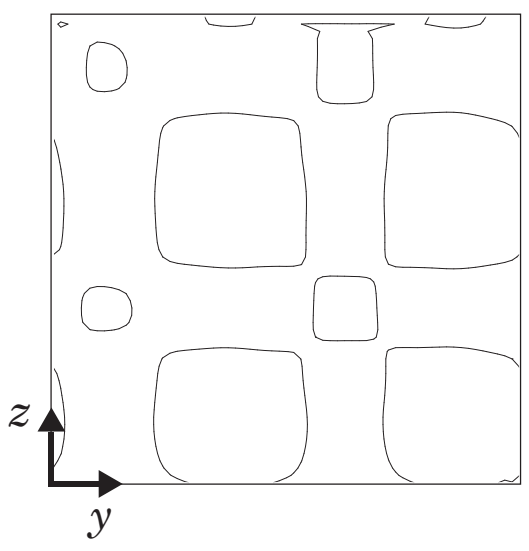

Fig. 9. Velocity vectors (left) and shear rate distribution (right) of Newtonian fluid for $\Delta p=1 \times 10^{-3}$ : (a) on $y / L=0.375$; (b) on $z / L=0.375$; (c) on $x / L=0.375$. $u_{0}$ is the superficial velocity. Contour interval of shear rate is $3.05 \times 10^{-5}$. 


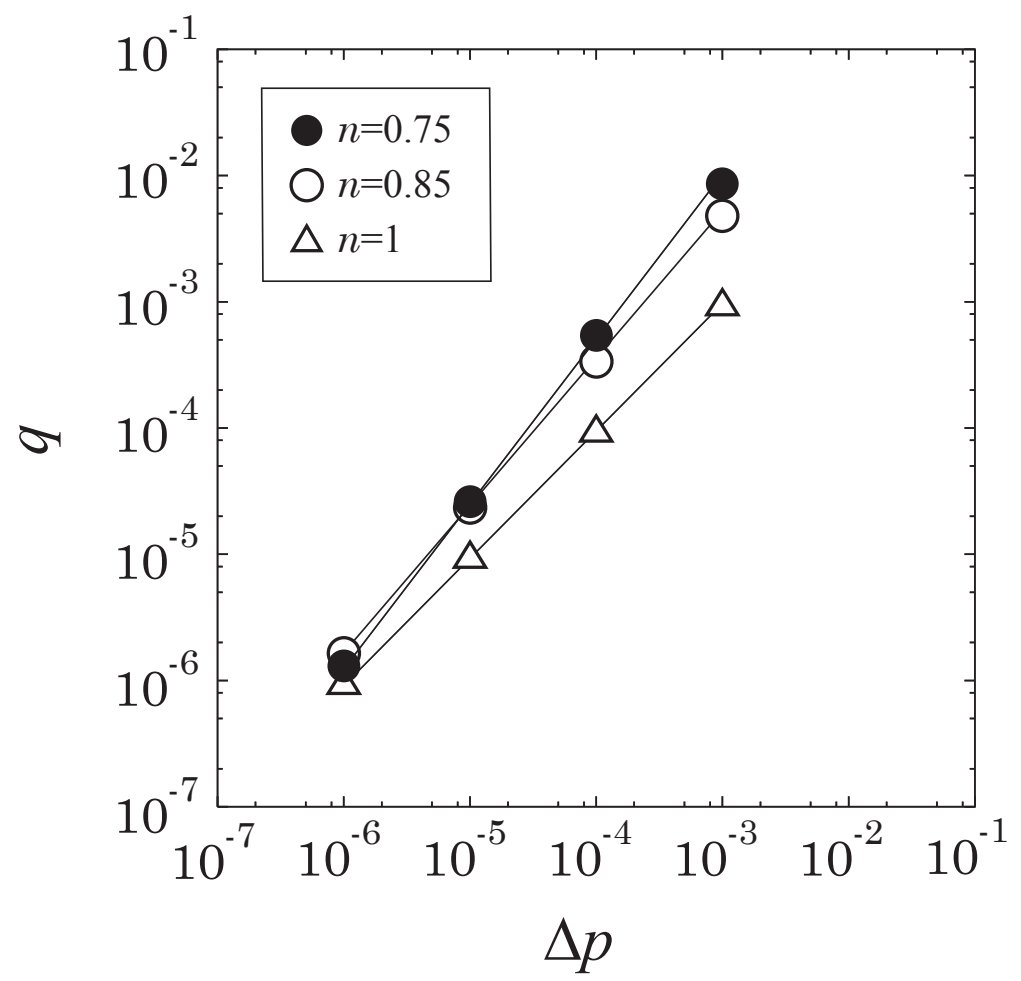

Fig. 10. Relation between pressure drops and fluid flux of flows in three-dimensional porous structure. 